\title{
Determinants of COVID-19-related lethality in multiple sclerosis: a meta-regression of observational studies
}

\author{
Luca Prosperini $^{1}\left[\right.$ Carla Tortorella ${ }^{1} \cdot$ Shalom Haggiag ${ }^{1} \cdot$ Serena Ruggieri ${ }^{2,3} \cdot$ Simonetta Galgani $^{1}$. \\ Claudio Gasperini ${ }^{1}$
}

Received: 18 November 2021 / Revised: 23 December 2021 / Accepted: 24 December 2021 / Published online: 4 January 2022

(c) The Author(s), under exclusive licence to Springer-Verlag GmbH Germany 2022

\begin{abstract}
Objective To identify risk factors for an increased lethality of COVID-19 in patients with multiple sclerosis (MS).

Methods We searched scientific databases to identify cohort studies with the number of deaths in patients with MS. We fitted inverse-variance weighted meta-regressions with random-effects models to identify potential moderators (determinants) of COVID-19-related lethality (outcome).

Results After an independent screening, 18 articles satisfied the eligibility criteria; all data were collected before anti-SARSCOV-2 vaccination was available. Out of 5,634 patients, 111 died, yielding a pooled death rate of $1.97 \%$ (95\% confidence intervals 1.61-2.33). There was a substantial heterogeneity between the included studies $\left(Q_{17}=66.9, p<0.001 ; I^{2}=77.5 \%\right)$, but no relevant publication bias $(p=0.085)$. Higher lethality was observed in studies including older patients $(\beta=0.80$, $p=0.025)$ and in studies with higher proportions of patients with comorbidity $(\beta=0.17, p=0.046)$, progressive disease course $(\beta=0.15, p=0.027)$, and current treatment with anti-CD20 agents $(\beta=0.18, p<0.001)$. Otherwise, higher proportions of patients treated with interferon beta $(\beta=-0.16, p<0.001)$ and teriflunomide $(\beta=-0.11, p=0.035)$ were associated with lower lethality. These estimates did not change even in both multivariable meta-regressions including adjustment variables and leave-one-out sensitivity analyses.

Conclusion Except for age and comorbidities, risk factors in common with the general population, we identified MS-specific determinants influencing the lethality of COVID-19. Our findings suggest the implementation of a risk mitigation plan for patients with progressive MS and for those treated with anti-CD20 agents.
\end{abstract}

Keywords Multiple sclerosis $\cdot$ COVID-19 $\cdot$ SARS-COV-2

Luca Prosperini

luca.prosperini@gmail.com

Carla Tortorella

carla.tortorella@gmail.com

Shalom Haggiag

lvshalom@hotmail.com

Serena Ruggieri

serena.ruggieri@gmail.com

Simonetta Galgani

sgalgani@ Scamilloforlanini.rm.it

Claudio Gasperini

c.gasperini@libero.it

1 Department of Neurology, S. Camillo-Forlanini Hospital, C.ne Gianicolense 87, 00152 Rome, Italy

2 Department of Human Neurosciences, Sapienza University, Viale dell'Università 30, 00185 Rome, Italy

3 Neuroimmunology Unit, Santa Lucia Foundation, Via del Fosso di Fiorano 64/65, 00143 Rome, Italy

\section{Introduction}

Multiple sclerosis (MS) is a long-lasting inflammatory and neurodegenerative disease of the central nervous system (CNS) representing the leading cause of progressive disability in young adults [1]. The long-term accumulation of severe disability is not strictly an immediate cause of death [2], nonetheless patients with MS are at higher risk of death than the general population, with an estimated reduction of life expectancy of approximately one decade [3]. This increased mortality risk is mainly attributable to complications arising from severe disability and longer disease duration, i.e. infections, cardiovascular or respiratory illnesses [3].

The growing availability of disease-modifying treatments (DMTs) has unquestionably improved the prognosis and possibly reduced the MS-related mortality; nevertheless, 
DMTs may increase the risk of infections [4], especially in older subjects [5]. This concern arose particularly following the current outbreak of the severe acute respiratory syndrome coronavirus-2 (SARS-COV-2) pandemic. The interplay between MS-related disability, DMT-induced alteration of immune homeostasis and the coronavirus disease-19 (COVID-19) may possibly result in a higher risk of morbidity and mortality in these patients [6]. Currently available data suggest an approximately $24 \%$-increased risk of death from COVID-19 in patients with MS than in the general population [7], mainly attributed to comorbidities and disease burden (i.e. progressive disease course and worse disability level $[8,9]$ ), whereas DMTs do not seem to affect survival $[10,11]$. However, treatment with anti-CD20 agents was shown as associated with an increased risk of hospitalization, while interferon beta was correlated with a reduced COVID-19 severity $[8,11,12]$.

In the attempt to disentangle this conflicting literature data, we performed a meta-regression of published studies to explore possible determinants of COVID-19-related death rate in patients with MS.

\section{Methods}

\section{Study design and registration}

We registered on PROSPERO (Registration Number: CRD42021246205) the review protocol before starting the meta-analysis with meta-regression and we applied the recommendations of the Preferred Reporting Items for Systematic Reviews and Meta-analyses (PRISMA) [13].

\section{Search strategy}

To identify studies on COVID-19 in patients with MS, we searched two medical (PubMed and Google Scholar) and one pre-print (MedRxiv) databases, using combinations of $\mathrm{MeSH}$ terms for articles published from January, the 1st 2020 until July 31, 2021, as follows: (multiple sclerosis) AND ((COVID-19) OR (coronavirus) OR (SARS-CoV-2)).

To develop our literature search strategy, we applied the PI(E)COS framework [14], as follows: Participants (P): people with multiple sclerosis; Intervention (I): not applicable; Exposure (E): COVID-19; Control (C): not applicable; Outcome $(\mathrm{O})$ : death due to COVID-19; Study designs or Settings (S): observational studies.

We selected only independent cohort studies, and excluded case series and case reports to minimize selection bias. To avoid the risk of missing relevant studies, we searched for additional articles through the bibliography of included ones and previously published reviews. Conference abstracts and articles written in languages other than English were not considered. To minimize the risk of duplicated data, we checked for authors and/or countries where data were collected. When two or more articles were from the same country, we included multiple studies only on the condition that there was no author overlap and data were collected in different sites. We excluded studies supported by Pharma Industries that were preferentially focused on just one DMT, to avoid both data overlap with independent observational cohorts and sponsorship bias [15].

To assess eligibility, two investigators (LP and $\mathrm{SH}$ ) independently searched for articles, and agreement between them was required to include an article; disagreement was solved by a third author after reading the whole article (CT).

\section{Study eligibility}

Each cohort study had to fulfil the following additional criteria to be included for quantitative synthesis: (i) patients must have a confirmed or suspected diagnosis of COVID-19; (ii) confirmed COVID-19 must be based on a laboratorybased assay through reverse transcriptase polymerase chain reaction (RT-PCR) on nasal and pharyngeal swabs, or by a positive serological test; (iii) suspected COVID-19 must be based on clinical and/or radiological features highly suggestive of SARS-COV-2 infection, even in absence of laboratory-based confirmation; (iv) the number of deaths observed over the entire follow-up must be clearly reported.

\section{Data extraction}

The list of the extracted variables for each study eligible for meta-regressions was pre-specified and included: first author; publication year; country; sample size; number of deaths (outcome of interest) and hospitalization attributable to COVID-19; proportion of patients with confirmed and suspected COVID-19; average age; proportion of male sex; proportion with comorbidity; average disability level expressed as expanded disability status scale (EDSS) score [16]; proportion with secondary or primary progressive disease course; proportion with recent steroid exposure (i.e. in the last 2 months before COVID-19 diagnosis); average lymphocyte count; proportion of untreated patients; proportion of patients under treatment with specific DMTs, i.e. anti-CD20 agents (ocrelizumab, ofatumumab and rituximab), interferon beta, dimethyl fumarate, glatiramer acetate, natalizumab, pulsed immune-reconstitution therapy (alemtuzumab and cladribine), sphingosine 1-phosphate receptor modulators (fingolimod, ozanimod, ponesimod, siponimod), teriflunomide. 


\section{Quality assessment}

The methodological quality of each included study was assessed by the Newcastle-Ottawa Scale (NOS) [17], which is a risk of bias assessment tool for observational studies that is recommended by the Cochrane Collaboration. The NOS consists of 8 items with 3 subscales, and the total maximum score of these 3 subsets is 9 . Three factors were considered to score the quality of included studies: (1) selection, including representativeness of the exposed cohort, selection of the non-exposed cohort, ascertainment of exposure, and demonstration that at the start of the study the outcome of interest was not present; (2) comparability, assessed on the basis of study design and analysis, and whether any confounding variables were adjusted for; and (3) outcome, based on the follow-up period and cohort retention, and ascertained by independent blind assessment, record linkage, or selfreport. By convention, a study scoring $\geq 6$ is considered a high-quality study.

\section{Statistical analysis}

We estimated the pooled effect size (ES) of included studies on lethality of COVID-19, i.e. the death rate calculated as number of deaths divided by the total sample size for each study. We applied the double arcsine transformation thatfor rare events such as the death-is more appropriate than other commonly used transformation. The double arcsine transformation can accommodate studies with zero events without requiring a continuity correction and has variancestabilizing property, as the variance depends only on the sample size without any correlation with the transformed proportion estimates [18]. Pooled estimates were weighted by inverse variance, thereby giving more weight to larger than smaller studies.

Between-study heterogeneity and inconsistency were expressed as Cochran's $Q$ and $I^{2}$, respectively. The Cochran's $Q$ is computed by summing the squared deviations of each study's ES from the pooled ES, weighting the contribution of each study by its inverse variance. The $\mathrm{I}^{2}$ was derived by the Cochran's $\mathrm{Q}$ according to the following formula: $(Q$ - degree of freedom $) / Q \times 100 \%$. We considered an $I^{2} \leq 40 \%$ as marginal, 30-60\% moderate and 50-90\% substantial heterogeneity, respectively (according to Cochrane Handbook for Systematic Reviews of Interventions, version 5.1.0; www.handbook-5-1.cochrane.org). Risk of publication bias was assessed by visual inspection of contour-enhanced funnel plot and the Egger test of asymmetry.

To account for the expected intra- and inter-study heterogeneity, meta-regression equations were fitted as inversevariance weighted random-effects models based on an empirical Bayes variance estimate [19].
We inserted in univariate meta-regression equations all variables (moderators) hypothetically influencing the pooled ES (see also the data extraction paragraph for the full list of moderators). All moderators were $\log (X+1)$-transformed to reduce the possibility of highly skewed variables potentially violating the normal distribution assumption. Normality assumption was then checked for all variables entered in models and for their residuals. To make easier the interpretation of metaregression results, we provided bubble plots for relevant associations. We also ran multivariable meta-regressions including adjustment variables to confirm findings from univariate analyses. Moreover, we conducted subgroup analyses to further validate meta-regression findings; subgroups were defined by median-split stratification of moderators significantly associated with lethality of COVID-19. Finally, leave-one-out sensitivity analyses were conducted by iteratively removing one article at a time to confirm that meta-regression results were not driven by any single study.

Two-tailed $p$ values less than 0.05 were considered significant. Data were analyzed by using the freeware software JASP version 0.12.1 (JASP Team, 2020; www.jasp-stats. org).

\section{Results}

\section{Search findings}

Our search retrieved 525 articles; after removing duplicates and screening for title and abstract, we assessed 140 full-text articles for eligibility. Of these, 18 articles met the eligibility criteria [20-37]. See Fig. 1 for the study selection process.

\section{Study characteristics}

Most of the data were collected during the first pandemic wave, and anyway, before the anti-SARS-COV-2 vaccination was available. According to different articles, data collection was locked in April 2020 [32], May 2020 [23, 27, 31, 33], June 2020 [22, 24, 25, 28, 30], whereas data of the remaining studies were collected until September 2020 [35, 36], October 2020 [20], December 2020 [26, 34], February 2021 [21, 37], April 2021 [29].

Studies were conducted in the following countries: Iran (3 articles) [23, 24, 33], United States (2 articles) [27, 32], Austria [26], Brazil [25], Chile [28], Czechia [37], France [31], Italy [36], Netherland [30], Poland [29], Saudi Arabia [21], Spain [22], Turkey [35]; two articles included data from different countries of Latin America [20] and North America [34]. Three articles included also data of 20 patients with 
Fig. 1 PRISMA flow-chart for study selection

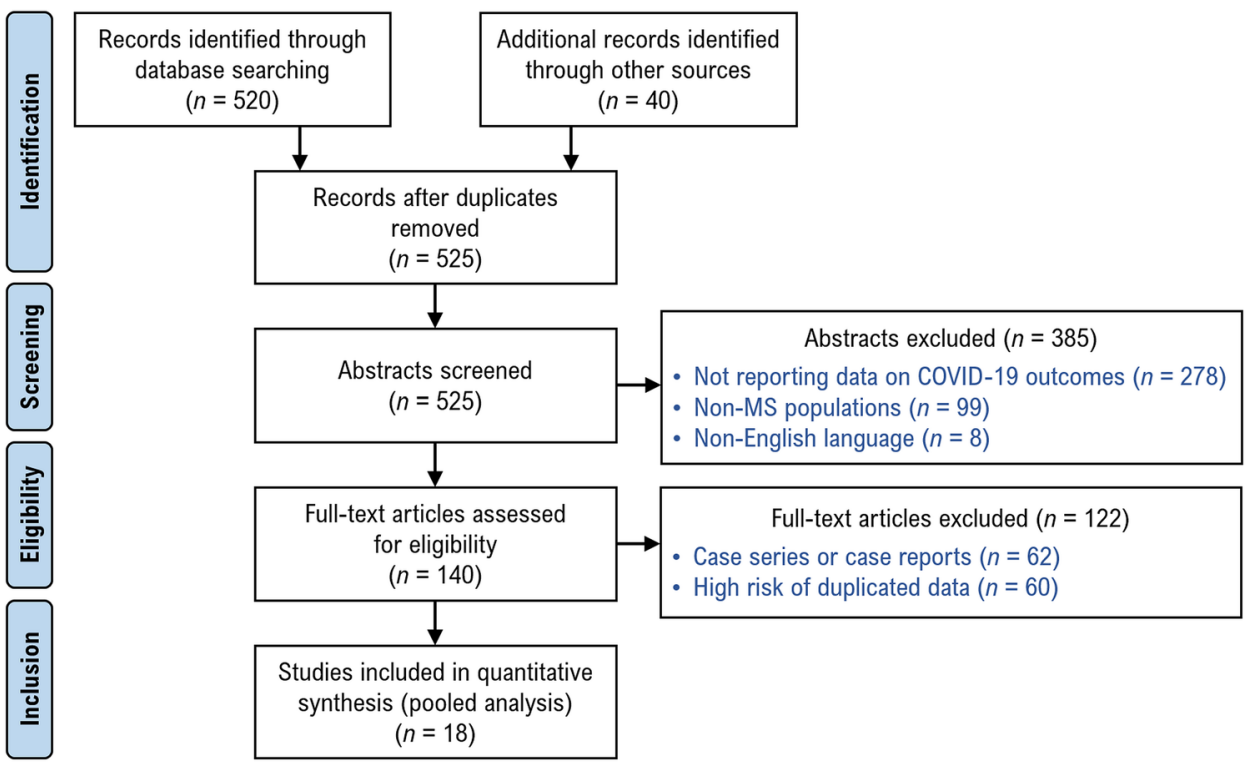

neuromyelitis optica spectrum disorder that were removed from further analysis [20, 28, 37].

All included studies were of good methodologic quality in terms of selection and outcome assessment, whereas slighter quality occurred in studies with smaller sample size (Table 1).

\section{Participants}

The pooled data extracted from 18 articles consisted in 5634 patients (see the Table 2 for the main characteristics of included cohorts). Their main age was 41.8 years and $1590(28.6 \%)$ of them were males (data on sex ratio were
Table 1 Assessment of methodological quality of included studies by the Newcastle-Ottawa Scale [17] of included studies

\begin{tabular}{|c|c|c|c|c|c|c|c|c|c|c|}
\hline \multirow[t]{2}{*}{ First Author } & \multicolumn{4}{|c|}{ Selection } & \multicolumn{2}{|c|}{ Comparability } & \multicolumn{3}{|c|}{ Outcome } & \multirow{2}{*}{$\begin{array}{l}\text { Total } \\
\text { quality } \\
\text { score }\end{array}$} \\
\hline & 1 & 2 & 3 & 4 & 1 & 2 & 1 & 2 & 3 & \\
\hline Alonso [20] & $\star$ & & $\star$ & $\star$ & & & $\star$ & $\star$ & $\star$ & 6 \\
\hline Alshamrani [21] & $\star$ & & $\star$ & $\star$ & & & $\star$ & $\star$ & $\star$ & 6 \\
\hline Arrambide [22] & $\star$ & & $\star$ & $\star$ & $\star$ & $\star$ & $\star$ & $\star$ & $\star$ & 8 \\
\hline Barzegar [23] & $\star$ & & $\star$ & $\star$ & & & $\star$ & $\star$ & $\star$ & 6 \\
\hline Bayat [24] & $\star$ & & $\star$ & $\star$ & & & $\star$ & $\star$ & $\star$ & 6 \\
\hline Brum [25] & $\star$ & & $\star$ & $\star$ & & & $\star$ & $\star$ & $\star$ & 6 \\
\hline Bsteh [26] & $\star$ & & $\star$ & $\star$ & $\star$ & $\star$ & $\star$ & $\star$ & $\star$ & 8 \\
\hline Chaudhry [27] & $\star$ & & $\star$ & $\star$ & & & $\star$ & $\star$ & $\star$ & 6 \\
\hline Ciampi [28] & $\star$ & & $\star$ & $\star$ & & & $\star$ & $\star$ & $\star$ & 6 \\
\hline Czarnowska [29] & $\star$ & & $\star$ & $\star$ & & & $\star$ & $\star$ & $\star$ & 6 \\
\hline Loonstra [30] & $\star$ & & $\star$ & $\star$ & & & $\star$ & $\star$ & $\star$ & 6 \\
\hline Louapre [31] & $\star$ & & $\star$ & $\star$ & $\star$ & $\star$ & $\star$ & $\star$ & $\star$ & 8 \\
\hline Parrotta [32] & $\star$ & & $\star$ & $\star$ & & & $\star$ & $\star$ & $\star$ & 6 \\
\hline Sahraian [33] & $\star$ & & $\star$ & $\star$ & & & $\star$ & $\star$ & $\star$ & 6 \\
\hline Salter [34] & $\star$ & & $\star$ & $\star$ & $\star$ & $\star$ & $\star$ & $\star$ & $\star$ & 6 \\
\hline Sen [35] & $\star$ & & $\star$ & $\star$ & $\star$ & $\star$ & $\star$ & $\star$ & $\star$ & 8 \\
\hline Sormani [36] & $\star$ & & $\star$ & $\star$ & $\star$ & $\star$ & $\star$ & $\star$ & $\star$ & 8 \\
\hline Stastna [37] & $\star$ & & $\star$ & $\star$ & $\star$ & $\star$ & $\star$ & $\star$ & $\star$ & 8 \\
\hline
\end{tabular}

Selection: 1. Representativeness of exposed cohort; 2. Selection of non-exposed cohort; 3. Ascertainment of exposure; 4. Demonstration that outcome of interest was not present at start of study; Comparability: 1. Adjust for the most important risk factors; 2. Adjust for other risk factors; Outcome: 1. Assessment of outcome; 2 . Follow-up length; 3 . Loss to follow-up rate. One " $\star "$ means 1 point 


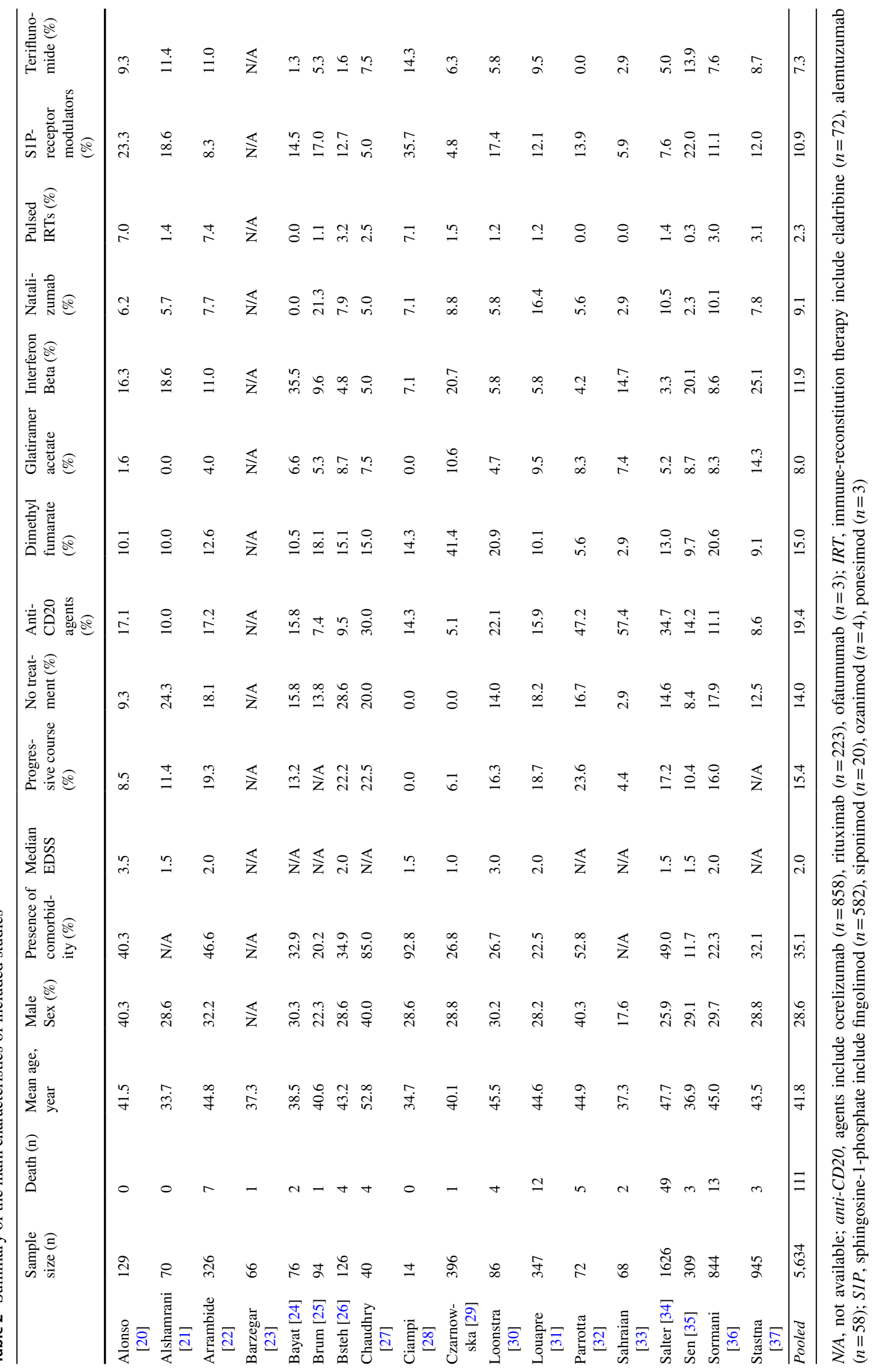


not available in one article [23]); 1,980 out 5,430 (35.1\%) had one or more comorbidity (data not available in 3 articles [21, 23, 33]); their median EDSS score was 2.0 (data not available in 7 articles [23-25, 27, 32, 33, 37]); 699 out of $4,529(15.4 \%)$ had a primary or secondary progressive disease course (data not available in 3 articles [23, 25, 37]).

Overall, 3,968 (70.4\%) patients were diagnosed with COVID-19 by a positive RT-PCR on nasal and/or pharyngeal swabs, while in the remaining 1,666 (29.6\%) the diagnosis was based on clinical and/or radiological suspicion. A total of 873 (15.5\%) patients required hospitalization.

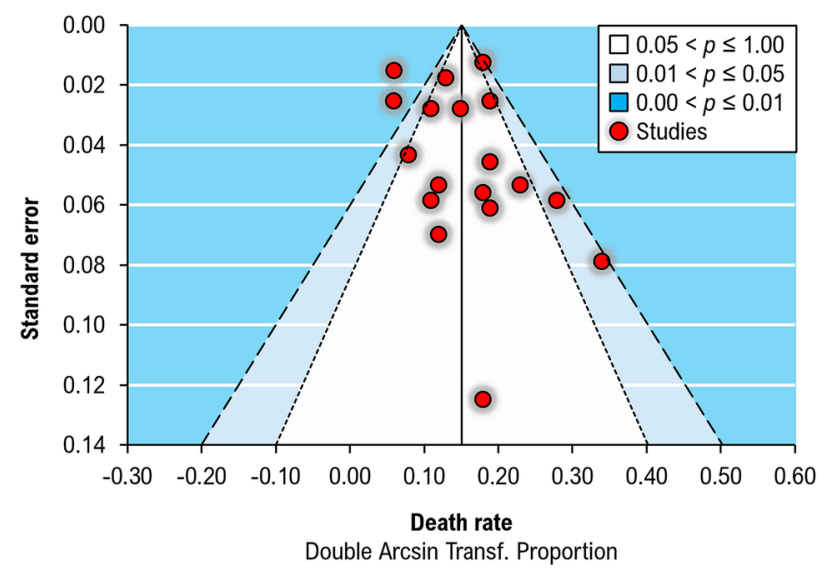

Fig. 2 Contour-enhanced funnel plot of included studies. For each study, the effect size (on $x$-axis) is plotted against the standard error (on $y$-axis), so that larger studies are placed towards the top and smaller studies more widely at the bottom

\section{Effect size (lethality of COVID-19)}

In the pooled cohort, there were a total of 111 deaths due to COVID-19, yielding a pooled death rate of $1.97 \%$ (95\% confidence intervals 1.61-2.33). As expected, there was a substantial heterogeneity between the included studies $\left(Q_{17}=66.9, p<0.001 ; I^{2}=77.5 \%\right)$.

The visual inspection of funnel plot (Fig. 2) showed that 16 out of 18 studies are in non-significant areas, with only minimal asymmetry across the included studies, thereby indicating a low probability of publication bias. This was also confirmed by the Egger test $(Z=1.72 ; p=0.085)$. Only two studies reporting an unexpected small number of deaths (in consideration of their sample size) are in areas of statistical significance [29, 37].

\section{Meta-regressions (univariate models)}

The overall results of meta-regression equations are shown in Table 3; the normality assumption was met for all moderators included in meta-regression models and their residuals (Appendix, Suppl. Table 1). We found no effect of world regions (America, Asia, and Europe) on lethality ( $p>0.3)$.

We found a positive correlation between lethality of COVID-19 and mean age of study populations $(\beta=0.80$, $p=0.025)$, proportions of patients with any comorbidity $(\beta=0.17, p=0.046)$, progressive disease course $(\beta=0.15$, $p=0.027)$, treatment with anti-CD20 agents $(\beta=0.18$, $p<0.001)$. By contrast, we found a negative correlation between lethality and proportions of patients under treatment with interferon beta $(\beta=-0.16, p<0.001)$ and teriflunomide
Table 3 Meta-regressions exploring the effect of demographic and clinical variables (moderators) on death rate due to COVID-19 in multiple sclerosis

\begin{tabular}{|c|c|c|c|c|c|c|c|c|}
\hline & \multirow[t]{2}{*}{$n$} & \multirow[t]{2}{*}{$\beta$} & \multirow[t]{2}{*}{ SE } & \multirow[t]{2}{*}{$p$ value } & \multicolumn{2}{|c|}{$95 \%$ confidence intervals } & \multirow[t]{2}{*}{$Q$} & \multirow[t]{2}{*}{$I^{2}$} \\
\hline & & & & & Lower bound & Upper bound & & \\
\hline Age & 18 & 0.798 & 0.356 & 0.025 & 0.100 & 1.496 & 51.2 & 71.9 \\
\hline Sex & 17 & 0.149 & 0.233 & 0.522 & -0.307 & 0.605 & 65.1 & 78.1 \\
\hline Comorbidity & 15 & 0.170 & 0.085 & 0.046 & 0.003 & 0.337 & 53.7 & 74.5 \\
\hline Progressive course & 15 & 0.148 & 0.064 & 0.027 & 0.097 & 0.375 & 25.9 & 68.2 \\
\hline EDSS & 11 & 0.182 & 0.171 & 0.288 & -0.154 & 0.518 & 27.6 & 64.4 \\
\hline Untreated & 17 & 0.062 & 0.041 & 0.131 & -0.019 & 0.144 & 54.3 & 74.6 \\
\hline Anti-CD20 agents & 17 & 0.176 & 0.044 & $<0.001$ & 0.089 & 0.263 & 26.4 & 43.2 \\
\hline Dimethyl fumarate & 17 & -0.088 & 0.075 & 0.239 & -0.237 & 0.059 & 64.8 & 76.1 \\
\hline Glatiramer acetate & 17 & -0.016 & 0.062 & 0.799 & -0.138 & 0.106 & 53.1 & 78.2 \\
\hline Interferon beta & 17 & -0.157 & 0.039 & $<0.001$ & -0.233 & -0.081 & 23.3 & 41.9 \\
\hline Natalizumab & 17 & -0.026 & 0.062 & 0.673 & -0.147 & 0.095 & 66.1 & 79.0 \\
\hline Pulsed IRT & 17 & -0.055 & 0.059 & 0.355 & -0.170 & 0.060 & 60.0 & 77.4 \\
\hline S1P-receptor modulators & 17 & -0.026 & 0.090 & 0.774 & -0.202 & 0.150 & 65.7 & 78.4 \\
\hline Teriflunomide & 17 & -0.115 & 0.054 & 0.035 & -0.221 & -0.008 & 50.2 & 70.6 \\
\hline
\end{tabular}

Anti-CD20, agents include ocrelizumab, ofatumumab, rituximab; IRT, immune-reconstitution therapy (alemtuzumab and cladribine); S1P, sphingosine-1-phosphate (fingolimod, ozanimod, ponesimod, siponimod); $S E$, standard error

Bold indicates significant two-tailed $p$-values $<0.05$ 
$(\beta=-0.11, p=0.035)$. Figure. 3 shows bubble plots for these relevant associations, suggesting no influence of study sizes on meta-regression findings.

\section{Meta-regressions (multi-variable models)}

Multivariable meta-regressions by including two covariates in single analyses showed no effect of age and progressive disease course $(p>0.2)$, as well as no effect of age and comorbidities $(p>0.2)$ on lethality of COVID-19, thus suggesting that these variables were dependent on each other. Otherwise, comorbidities and progressive disease course $(p<0.05)$ were independently associated with the lethality even when entered together in meta-regression equation. As few data were available on average lymphocyte count and proportion of patients with recent steroid exposure, we did not perform further analysis on these two variables.

The significant associations observed between lethality and specific DMTs survived even in multivariable meta-regressions including age and progressive disease course as adjustment variables: anti-CD20 agents $(\beta=0.13$, $p=0.011)$, interferon beta $(\beta=-0.14, p=0.038)$ and teriflunomide $(\beta=-0.10, p=0.047)$; similar estimates were found even including other combination of adjustment variables (data not shown). This indicates that the effect of antiCD20 agents (detrimental), as well as interferon beta and teriflunomide (favourable) on lethality was not influenced by the intrinsic characteristics of analysed cohorts.

We failed to identify any additive effect on lethality of the interaction of anti-CD20 agents with age and/or comorbidity (data not shown).

\section{Subgroup and sensitivity analyses}

To validate meta-regression findings, we performed subgroup analyses on moderators significantly associated with lethality of COVID-19. We found significant subgroup effects ( $p$ values $<0.05$ ) indicating increased lethality in study cohorts with older age (on average $>43$ years), greater proportions $(>13 \%)$ of patients with progressive disease course and current treatment with anti-CD20 agents $(>15 \%)$. On the other hand, we found reduced lethality $(p=0.007)$ in the subgroup with greater proportions of patients under interferon beta treatment $(>10 \%)$. No effect was found in subgroups identified after median-split of proportions with comorbidity and current treatment with teriflunomide ( $p$ values $>0.1$ ). Figure 4 shows a graphical representation of subgroup analyses.

The leave-one-out analyses confirmed that estimates of univariate meta-regressions did not change substantially even after iteratively removing one study at a time,


Fig. 3 Bubble plots of relevant association between COVID-19 lethality (effect size expressed as double arcsine-transformed death rate) and demographic, clinical and treatment variables (moderators) in observational studies on multiple sclerosis; each circle represents a study, with the circle area proportional to the sample size of that study 




Fig. 4 Subgroup analyses on moderators significantly associated with COVID-19-related lethality (effect size expressed as double arcsinetransformed death rate) in univariate meta-regressions. The black diamond represents the pooled effect size, whereas the white diamonds represent the effect size for each subgroup, as defined by median-split stratification of moderators. Percentages indicate the median age and proportions of patients in each subgroup

indicating that the pooled results were not driven by any single study (Appendix, Suppl. Table 2).

\section{Discussion}

The aim of this study was to identify risk factors associated with an increased lethality of COVID-19 in patients with MS. We fitted meta-regression analyses of observational studies published so far, paying attention to avoid data duplication, and by handling data in the more appropriate way to deal with a rare event such as death (i.e. double arcsine transformation) [18].

We estimated a pooled death rate of $1.97 \%$, a slightly lower proportion as compared with a published systematic review reporting a $3.0 \%$ death rate among patients with suspected/confirmed COVID-19 cases [9]. However, this latter review included also conference abstracts, case report and case series [9], instead we choose to not include articles other than observational cohort studies to prevent the risk data duplication.

Other than age and presence of comorbidities-which are two known risk factors shared with the general population [38] —we identified several MS-specific determinants influencing the lethality of COVID-19, namely a progressive disease course and current treatment with different DMTs (anti-CD20 agents, interferon beta, teriflunomide).

Notably, male sex and EDSS score did not associate with higher death rate, unlike prior studies $[12,39]$. The low variability both in proportions of males (interquartile range $28-31 \%$ ) and in median EDSS score (ranging from 1.0 to 3.5) among the included cohorts may, at least partially, explain this discrepancy. Moreover, in contrast with prior studies, our analysis did not show an increased risk of severe or fatal COVID-19 among untreated patients [31,36], neither a decreased risk among those treated with fumarates and natalizumab [34].

\section{MS-specific determinants of death risk of COVID-19}

Patients with progressive disease course and worse disability level have an increased risk of severe or fatal COVID-19 [9, 11] that is in line with previous observations on infections in general, especially those affecting the respiratory tracts [40-42].

Furthermore, our data indicate a diverse effect on lethality of COVID-19 from different DMTs used in MS. Anti-CD20 agents showed a detrimental effect, while interferon beta and teriflunomide had a protective effect on the death risk.

Prolonged anti-CD20 treatment has been associated with hypogammaglobulinemia and impaired B-cell reconstitution that, in turn, may lead to increased infection risk [43], regardless of the underlying pathological condition. In a large cohort study, patients with MS treated with rituximab experienced a higher rate of serious infections than those under other DMTs [44]. Pivotal phase III trials showed a higher incidence of respiratory tract infections in ocrelizumab arm than placebo arm and interferon beta arm in patients with primary progressive and relapsing-remitting, respectively [45].

Several smaller studies included in our analysis did not document any significant effect of anti-CD20 agents on COVID-19 severity [20, 22, 26, 27, 29, 30, 32, 33], whilst the three largest studies showed a significant association [34, $36,37]$. Other two large studies (not included in our metaregression to prevent data duplication), one combining data from Italy and France [39] and the other one aggregating data from 12 data-sources in 28 different countries [11], confirmed an increased risk for severe COVID-19 in patients treated with anti-CD20 agents. However, these studies were inconclusive concerning the risk of death from COVID-19, probably due to low statistical power to explore rare events.

Interestingly, anti-CD20 agents were the most prevalent DMTs, not only in the pooled cohort (almost 20\%), but also in many of the included studies [20, 22, 27, 30-37]. While one can argue about the possibility of selection or reporting bias, another plausible explanation might 
encompass an increased susceptibility to SARS-COV-2 infections due to current treatment with anti-CD20 agents [12].

The protective role of interferon beta on the death risk from COVID-19 is not surprising. Type I interferons are abundantly secreted in response to viral infections, and for this reason they are considered as a potential treatment for COVID-19 [46]. Individuals exhibiting low type I interferon secretion capacity are more vulnerable to SARS-COV-2 infection and life-threatening COVID-19 pneumonia [47]. Other than inborn errors, autoimmune diseases (including MS) can be linked with altered production of type I interferons [48]. Of note, a lower risk of pneumonia has been observed in patients currently treated with interferon beta in a large cohort of MS cases followed for an average time of 8.5 years [49].

The protective effect of teriflunomide on death rate, as by the present meta-regression, reinforces the putative antiviral role of inhibiting the pyrimidine biosynthesis to suppress transcription and replication of several viruses [50]. However, this latter finding should be interpreted with caution, as the antiviral role of teriflunomide is just hypothetical and, differently from interferon beta, not yet supported by clinical data.

\section{Limitations}

Despite the robust methodological and statistical approach, we are aware that our study has some limitations.

First, the clinical and statistical heterogeneity of the samples, resulting from pooling data from mixed MS cohorts.

Second, we included observational studies that, for their intrinsic nature, suffer from limited internal validity as affected by hidden confounding factors and reporting bias [51], as for example in regard to the aforementioned high prevalence of patients treated with anti-CD20 agents.

Third, we should consider the availability bias due to the absence of some data, thus forcing us to reduce the pooled sample size when analyzing several variables (comorbidities, EDSS score, progressive course) and not to fit several meta-regressions exploring the death risk in relation with lymphocyte count, recent steroid exposure, timing from the last anti-CD20 administration, and timing from pulsed immune-reconstitution therapy with alemtuzumab or cladribine.

Lastly, when interpreting our meta-regressions, we should take into account the ecological fallacy of such an approach [52], considering that the association found in pooled data does not necessarily reflect the association found in individual patient data reported by cohort studies included in our analyses [20-37]. Nevertheless, our findings appear biologically and clinically plausible, in line with those from larger observational studies [11, 34, 36, 37, 39].

\section{Clinical implications}

Despite the aforementioned limitations, we believe our study may have relevant clinical implications. Clinicians and patients with MS and should be made aware of a possible increased lethality of COVID-19 in case of advancing age, presence of comorbidity, and progressive disease course.

Risk mitigation plans should be implemented in patients treated with anti-CD20 agents, also considering the recent report of a reduced humoral response to anti-COVID-19 vaccination in patients receiving ocrelizumab [53,54]. When applicable, ocrelizumab and rituximab should be started after anti-COVID-19 vaccination, unless the risk:benefit ratio supports the need for an urgent treatment [55]. In patients already on treatment with anti-CD20 agents, the timing of vaccination should be scheduled with a delay of at least 3 months from the last administration, according to the most recent recommendations [55]. Interferon beta, and to a lesser extent, teriflunomide, should be considered among the first treatment options in older patients with comorbidity who require lower-efficacy DMTs [56]. Notably, all the aforementioned suggestions came from expert consensus rather than clinical evidence, therefore they should be interpreted with caution and cannot be considered as guidelines until validation by 'ad hoc' studies.

\section{Conclusions}

The present study extends findings from our recently published pooled analysis of observational studies where we found a $24 \%$-increased risk of death from COVID-19 in patients with MS by an indirect standardization method (using as reference the age-specific case-fatality rates of COVID-19 in general population obtained from the WHO website) [7].

In these meta-regressions of observational studies, we identified clinical determinants for an increased lethality of COVID-19 in patients with MS. These determinants include age, presence of comorbidity, progressive disease course, and current treatment with anti-CD20 agents. Interferon beta and teriflunomide were associated with a reduced lethality, thus suggesting their protective role.

We hope that these findings, despite their intrinsic and overwhelming limitations, may help neurologists in optimizing the monitoring and treatment decision-making processes in this global crisis due to the pandemic COVID-19 
outbreak. Future investigation is warranted to determine if the introduction of anti-SARS-COV-2 vaccines would alter our findings regarding both the increased death risk and lethality determinants of COVID-19.

Supplementary Information The online version contains supplementary material available at https://doi.org/10.1007/s00415-021-10951-6.

Funding LP: consulting fees and/or speaker honoraria from Biogen, Celgene, Genzyme, Merck-Serono, Novartis and Teva; travel grants from Biogen, Genzyme, Novartis and Teva; research grants from the Italian MS Society (Associazione Italiana Sclerosi Multipla) and Genzyme. CT: honoraria for speaking and travel grants from Biogen, Sanofi-Aventis, Merck Serono, Bayer-Schering, Teva, Genzyme, Almirall and Novartis. SH: travel funding and/or speaker honoraria from Biogen, Roche, Genzyme, Novartis, CSL Behring. SR: personal fees and non-financial support from Biogen, Genzyme, Merck-Serono, Novartis, and Teva. SG: honoraria for speaking and travel grants from Biogen, Sanofi-Aventis, Merck Serono, Bayer-Schering, Teva, Genzyme, Almirall and Novartis. CG: fees as invited speaker or travel expenses for attending meeting from Biogen, Merck-Serono, Teva, Sanofi, Novartis, Genzyme.

Data availability statement The data that support the findings of this study are available from the corresponding author (luca.prosperini@ gmail.com) upon reasonable request.

\section{Declarations}

Conflicts of interest Nothing to report relevant to the present study.

Ethics approval Not applicable.

\section{References}

1. Thompson AJ, Baranzini SE, Geurts J, Hemmer B, Ciccarelli O (2018) Multiple sclerosis. Lancet 391(10130):1622-1636

2. Scalfari A, Knappertz V, Cutter G et al (2013) Mortality in patients with multiple sclerosis. Neurology 81(2):184-192

3. Ragonese P, Aridon P, Salemi G, D'Amelio M, Savettieri G (2008) Mortality in multiple sclerosis: a review. Eur J Neurol 15(2):123-127

4. Grebenciucova E, Pruitt A (2017) Infections in patients receiving multiple sclerosis disease-modifying therapies. Curr Neurol Neurosci Rep 17(11):88

5. Grebenciucova E, Berger JR (2017) Immunosenescence: the role of aging in the predisposition to neuro-infectious complications arising from the treatment of multiple sclerosis. Curr Neurol Neurosci Rep 17(8):61

6. Hartung H-P, Aktas O (2020) COVID-19 and management of neuroimmunological disorders. Nat Rev Neurol 16(7):347-348

7. Prosperini L, Tortorella C, Haggiag S et al (2021) Increased risk of death from COVID-19 in multiple sclerosis: a pooled analysis of observational studies. J Neurol. https://doi.org/10. 1007/s00415-021-10803-3

8. Sormani MP, Schiavetti I, Carmisciano L et al (2021) COVID19 severity in multiple sclerosis: putting data into context. Neurol Neuroimmunol Neuroinflamm 9(1):e1105. https://doi.org/ 10.1212/NXI.0000000000001105
9. Barzegar M, Mirmosayyeb O, Gajarzadeh M et al (2021) COVID-19 among patients with multiple sclerosis: a systematic review. Neurol Neuroimmunol Neuroinflammation 8(4):e1001

10. Moreno-Torres I, Meca Lallana V et al (2021) Risk and outcomes of COVID-19 in patients with multiple sclerosis. Eur J Neurol 28(11):3712-3721

11. Simpson-Yap S, Brouwer ED, Kalincik T et al (2021) Associations of DMT therapies with COVID-19 severity in multiple sclerosis. Neurology. https://doi.org/10.1212/WNL.0000000000012753

12. Reder AT, Centonze D, Naylor ML et al (2021) COVID-19 in patients with multiple sclerosis: associations with disease-modifying therapies. CNS Drugs 35(3):317-330

13. Moher D, Liberati A, Tetzlaff J, Altman DG, PRISMA Group (2009) Preferred reporting items for systematic reviews and metaanalyses: the PRISMA Statement. Open Med Peer-Rev Indep Open-Access J 3(3):e123-130

14. O’Sullivan D, Wilk S, Michalowski W, Farion K (2013) Using PICO to align medical evidence with MDs decision making models. Stud Health Technol Inform 192:1057

15. Lundh A, Lexchin J, Mintzes B, Schroll JB, Bero L (2017) Industry sponsorship and research outcome. Cochrane Database Syst Rev 2(2):MR000033.

16. Kurtzke JF (1983) Rating neurologic impairment in multiple sclerosis: an expanded disability status scale (EDSS). Neurology 33(11):1444-1452

17. Stang A (2010) Critical evaluation of the Newcastle-Ottawa scale for the assessment of the quality of nonrandomized studies in meta-analyses. Eur J Epidemiol 25(9):603-605

18. Lin L, Xu C (2020) Arcsine-based transformations for meta-analysis of proportions: pros, cons, and alternatives. Rep Health Sci. https://doi.org/10.1002/hsr2.178

19. Sidik K, Jonkman JN (2007) A comparison of heterogeneity variance estimators in combining results of studies. Stat Med 26(9):1964-1981

20. Alonso R, Silva B, Garcea O et al (2021) COVID-19 in multiple sclerosis and neuromyelitis optica spectrum disorder patients in Latin America. Mult Scler Relat Disord 51:102886

21. Alshamrani F, Alnajashi H, AlJumah M et al (2021) Registry of patients with multiple sclerosis an COVID-19 infection in Saudi Arabia. Mult Scler Relat Disord 52:103004

22. Arrambide G, Llaneza-González MÁ, Costa-Frossard França L et al (2021) SARS-CoV-2 infection in multiple sclerosis: results of the Spanish Neurology Society Registry. Neurol Neuroimmunol Neuroinflam 8:e1024

23. Barzegar M, Mirmosayyeb O, Ghajarzadeh M et al (2020) Characteristics of COVID-19 disease in multiple sclerosis patients. Mult Scler Relat Disord 45:102276

24. Bayat M, Fayyazpoor A, Haghighi AB et al (2020) SARS-CoV-2 infection among patients with multiple sclerosis, a cross-sectional study. MedRxiv. https://doi.org/10.1101/2020.10.17.20214429

25. Brum DG et al. Neuroimmunology Brazilian Study Group Focused on COVID-19 and MS (2021) Incidence and clinical outcome of Coronavirus disease 2019 in a cohort of 11,560 Brazilian patients with multiple sclerosis. Mult Scler.https://doi.org/ $10.1177 / 1352458520978354$.

26. Bsteh G, Assar H, Hegen H et al (2021) COVID-19 severity and mortality in multiple sclerosis are not associated with immunotherapy: insights from a nation-wide Austrian registry. PLoS ONE 16:e0255316

27. Chaudhry F, Bulka H, Rathnam AS et al (2020) COVID-19 in multiple sclerosis patients and risk factors for severe infection. $\mathrm{J}$ Neurol Sci 418:117147

28. Ciampi E, Uribe-San-Martín R, Soler B et al (2020) COVID-19 in MS and NMOSD: a multicentric online national survey in Chile. Mult Scler Relat Disord 45:102392 
29. Czarnowska A, Brola W, Zajkowska O et al (2021) Clinical course and outcome of SARS-CoV-2 infection in multiple sclerosis patients treated with disease-modifying therapies-the Polish experience. Neurol Neurochir Pol 55:11

30. Loonstra FC, Hoitsma E, van Kempen ZL et al (2020) COVID19 in multiple sclerosis: the Dutch experience. Mult Scler 26:1256-1260

31. Louapre C, Collongues N, Stankoff B et al (2020) Clinical characteristics and outcomes in patients with coronavirus disease 2019 and multiple sclerosis. JAMA Neurol 77(9):1079-1088

32. Parrotta E, Kister I, Charvet L et al (2020) COVID-19 outcomes in MS: observational study of early experience from NYU multiple sclerosis comprehensive care center. Neurol Neuroimmunol Neuroinflam 7:e835

33. Sahraian MA, Azimi A, Navardi S, et al (2020) Evaluation of the rate of COVID-19 infection, hospitalization and death among Iranian patients with multiple sclerosis. Mult Scler Relat Disord 46:102472.

34. Salter A, Fox RJ, Newsome SD et al (2021) Outcomes and risk factors associated with SARS-CoV-2 infection in a north American registry of patients with multiple sclerosis. JAMA Neurol 78(6):699-708

35. Sen S, Karabudak R, Schiavetti I et al (2021) The outcome of a national MS-Covid-19 study: What the Turkish MS cohort reveals? Mult Scler Relat Disord 52:102968

36. Sormani MP, De Rossi N, Schiavetti I et al (2021) Disease-modifying therapies and coronavirus disease 2019 severity in multiple sclerosis. Ann Neurol 89:780-789

37. Stastna D, Menkyova I, Drahota J et al (2021) Multiple sclerosis, neuromyelitis optica spectrum disorder and COVID-19: a pandemic year in Czechia. Mult Scler Relat Disord 54:103104

38. Onder G, Rezza G, Brusaferro S (2020) Case-fatality rate and characteristics of patients dying in relation to COVID-19 in Italy. JAMA. Available at: https://jamanetwork.com/journals/jama/fulla rticle/2763667 [Accessed August 26, 2021].

39. Sormani MP, Salvetti M, Labauge P et al (2021) DMTs and Covid19 severity in MS: a pooled analysis from Italy and France. Ann Clin Transl Neurol 8(8):1738-1744

40. Montgomery S, Hillert J, Bahmanyar S (2013) Hospital admission due to infections in multiple sclerosis patients. Eur J Neurol 20(8):1153-1160

41. Nelson RE, Xie Y, DuVall SL et al (2015) Multiple sclerosis and risk of infection-related hospitalization and death in US veterans. Int J MS Care 17(5):221-230

42. Goodin DS, Ebers GC, Cutter G et al (2012) Cause of death in MS: long-term follow-up of a randomised cohort, 21 years after the start of the pivotal IFN $\beta-1 b$ study. BMJ Open 2(6):e001972
43. Sacco KA, Abraham RS (2018) Consequences of B-cell-depleting therapy: hypogammaglobulinemia and impaired B-cell reconstitution. Immunotherapy 10(8):713-728

44. Luna G, Alping P, Burman J et al (2020) Infection risks among patients with multiple sclerosis treated with fingolimod, natalizumab, rituximab, and injectable therapies. JAMA Neurol 77(2):184-191

45. Moiola L, Barcella V, Benatti S et al (2021) The risk of infection in patients with multiple sclerosis treated with diseasemodifying therapies: a Delphi consensus statement. Mult Scler 27(3):331-346

46. WHO Solidarity Trial Consortium, Pan H, Peto R et al (2021) Repurposed antiviral drugs for covid-19 - interim WHO solidarity trial results. N Engl J Med 384(6):497-511

47. Zhang Q, Bastard P, Liu Z, et al (2020) Inborn errors of type I IFN immunity in patients with life-threatening COVID-19. Science 370(6515):eabd4570.

48. Severa M, Farina C, Salvetti M, Coccia EM (2020) Three decades of interferon- $\beta$ in multiple sclerosis: can we repurpose this information for the management of SARS-CoV2 infection? Front Immunol 11:1459

49. Wijnands JMA, Zhu F, Kingwell E et al (2018) Disease-modifying drugs for multiple sclerosis and infection risk: a cohort study. J Neurol Neurosurg Psychiatry 89(10):1050-1056

50. Lucas-Hourani M, Dauzonne D, Jorda P et al (2013) Inhibition of pyrimidine biosynthesis pathway suppresses viral growth through innate immunity. PLoS Pathog 9(10):e1003678

51. Metelli S, Chaimani A (2020) Challenges in meta-analyses with observational studies. Evid Based Ment Health 23(2):83-87

52. Sedgwick P (2011) The ecological fallacy. BMJ. Available at: https://www.bmj.com/content/343/bmj.d4670 [Accessed May 3, 2020].

53. Achiron A, Mandel M, Dreyer-Alster S et al (2021) Humoral immune response to COVID-19 mRNA vaccine in patients with multiple sclerosis treated with high-efficacy disease-modifying therapies. Ther Adv Neurol Disord 14:17562864211012836

54. Tortorella C, Aiello A, Gasperini C, et al (2021) Humoral and T-cell specific immune responses to SARS-CoV-2 mRNA vaccination in patients with MS using different disease-modifying therapies. Neurology [Accepted for publication].

55. Centonze D, Rocca MA, Gasperini C et al (2021) Disease-modifying therapies and SARS-CoV-2 vaccination in multiple sclerosis: an expert consensus. J Neurol 268(11):3961-3968

56. Baker D, Amor S, Kang AS, Schmierer K, Giovannoni G (2020) The underpinning biology relating to multiple sclerosis disease modifying treatments during the COVID-19 pandemic. Mult Scler Relat. Disord. 43:102174 